Research Paper

\title{
Yangyin Fuzheng Decoction enhances anti-tumor efficacy of cisplatin on lung cancer
}

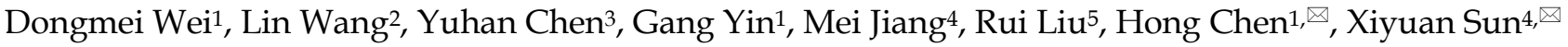 \\ 1. Department of Traditional Chinese Geriatric Medicine, The First Hospital of Qiqihar, Qiqihaer, 30 Gongyuan Road, Longsha District, Qiqihaer, \\ Heilongjiang, 161005, PR China. \\ 2. Department of Nursing, The First Hospital of Qiqihar, Qiqihaer, 30 Gongyuan Road, Longsha District, Qiqihaer, Heilongjiang, 161005, PR China. \\ 3. Department of Chinese and Western medicine combined with oncology, Academy of Traditional Chinese Medicine Guang'anmen Hospital, No.5, North \\ Line, Xicheng District, Beijing 100053 PR China. \\ 4. Department of Chinese and Western medicine combined with oncology, The First Hospital of Qiqihar, Qiqihaer, 30 Gongyuan Road, Longsha District, \\ Qiqihaer, Heilongjiang, 161005, PR China. \\ 5. Biobank of The First Hospital of Qiqihar, The First Hospital of Qiqihar, Qiqihaer, 30 Gongyuan Road, Longsha District, Qiqihaer, Heilongjiang, 161005, PR \\ China.
}

$\triangle$ Corresponding authors: Hong Chen, Department of Traditional Chinese Geriatric Medicine, The First Hospital of Qiqihar, Qiqihaer, 30 Gongyuan Road, Longsha District, Qiqihaer, Heilongjiang, 161005, PR China. Tel: 86-0452-2459559; E-mail: qqhedyyych@tj1998.com; Xiyuan Sun, Department of Chinese and Western medicine combined with oncology, The First Hospital of Qiqihar, Qiqihaer, 30 Gongyuan Road, Longsha District, Qiqihaer, Heilongjiang, 161005, PR China. Tel: 86-0452-2459427; E-mail: sunxiyuan2004@126.com

(C) Ivyspring International Publisher. This is an open access article distributed under the terms of the Creative Commons Attribution (CC BY-NC) license (https://creativecommons.org/licenses/by-nc/4.0/). See http://ivyspring.com/terms for full terms and conditions.

Received: 2017.12.23; Accepted: 2018.02.16; Published: 2018.04.12

\begin{abstract}
Traditional Chinese medicine has been widely used in cancer treatment in China. Yangyin Fuzheng Decoction is a traditional Chinese compound medicine, composed of 12 traditional Chinese herbs. This study aimed to investigate anti-tumor activity and the underlying mechanisms of Yangyin Fuzheng Decoction combined with cisplatin in the treatment of lung cancer. We established lung cancer model in C57BL/6 mice injected with mouse Lewis lung cancer cells. Our results demonstrated that Yangyin Fuzheng Decoction treatment increased necrotic area in tumor tissue, and significantly enhanced the recruitment of inflammatory cells into the tumor. In addition, Yangyin Fuzheng Decoction treatment enhanced the anti-tumor efficacy of cisplatin and partially recovered mouse body weight loss caused by cisplatin treatment. Mechanistically, we found that Yangyin Fuzheng Decoction upregulated the expression of pro-apoptotic proteins $\mathrm{p} 53$ and Bax and suppressed the expression of anti-apoptotic protein Bcl-2. Combined treatment of Yangyin Fuzheng Decoction and cisplatin further increased p53 and Bax levels and suppressed Bcl-2 level. Taken together, these data suggest that Yangyin Fuzheng Decoction could synergistically enhance the apoptotic signaling in cancer cells during chemotherapy. In addition, it has health improving and immune response enhancing effects. Yangyin Fuzheng Decoction could be a promising adjunct agent for lung cancer chemotherapy.
\end{abstract}

Key words: Yangyin Fuzheng Decoction; Lung cancer; Cisplatin; Chemotherapy; Chinese traditional medicine

\section{Introduction}

Cancer is a leading cause of death in China. Among all cancer cases, lung cancer is the most commonly diagnosed cancer in men and the second in women in China, and it is the leading cause of cancer-related death in both men and women [1]. Due to the increasing air pollution and large smoking population, it is predicted that lung cancer would persistently be a major public health problem in China
[1]. Therefore, effective lung cancer treatment strategy is in great need, especially for late stage lung cancer which relays on chemotherapy.

Despite the recent development of targeting anti-cancer drugs, cisplatin and its derivatives remain the first line chemotherapy drugs for lung cancer patients, especially for patients with advanced lung cancer [2-4]. Cisplatin is an alkylating agent that 
induces DNA damages, and it kills cancer cells by the activation of cell death pathways in fast growing cancer cells. Unfortunately, cisplatin also causes damages to normal cells in the body, especially normal active dividing cells such as lymphocytes and hair follicle cells. The strong side effects of cisplatin significantly impair the patient's survival and life quality, limiting its use in cancer patients with poor health condition.

To alleviate the adverse effects of chemotherapy, traditional Chinese medicines that can improve health condition and boost immune-system have been employed in combination with chemotherapy [5-7]. The traditional Chinese medicines showed significant benefits in cancer treatment by improving patient health after surgery, radiotherapy or chemotherapy. Shenqi Fuzheng Injection (SFJ) is one of these traditional Chinese medicines which has approved by China's State Food and Drug Administration [8]. SFJ is the extraction from two Chinese herbs, Radix codonopsis and Radix astragali, and is used to improve immune function, heart disease and multiple organ dysfunction in elderly people [9]. In recent years, SFJ has been widely used in combination with chemotherapy for lung, colorectal, gastric and liver cancers, and demonstrated synergetic anti-tumor effect in these trials with improved immune function and reduction of adverse events during chemotherapy [10, 11]. To further explode possible use of traditional Chinese medicines in cancer treatment, a traditional Chinese compound medicine, Yangyin Fuzheng Decoction (YFD) was utilized in this study. Yangyin Fuzheng Decoction is composed of 12 Chinese herbs, including Radix codonopsis and Radix astragali. Using lung cancer model in C57BL/ 6 mice implanted with mouse Lewis lung cancer cells, we evaluated the effects of YFD used alone or in combination with cisplatin on lung cancer therapy.

\section{Materials and Methods}

\section{Cell culture and agents}

Mouse Lewis lung cancer cells were provided by Boster Biological Technology (Wuhan, China) and cultured in DMEM medium supplemented with $10 \%$ fetal bovine serum (FBS) in a humid atmosphere with $5 \% \mathrm{CO}_{2}$ at $37^{\circ} \mathrm{C}$. Yangyin Fuzheng Decoction was obtained from Qiqihar No. 1 Hospital. IV grade cisplatin was from Qilu Pharmaceutical (Jinan, China).

\section{Animals}

C57BL/6 mouse (inbred strain, male, body weight $18-20 \mathrm{~g}$ ) was provided by Qingdao
Experimental Animal Center (Qingdao, China, Certification No. SCXK 2014-0001). All animal experiments were approved by Animal Care and Use Committee of The First Hospital of Qiqihar. Mouse Lewis lung cancer cells at concentration of $2 \times 10^{6} /$ $\mathrm{mL}$ was injected into mouse abdomen. After 9 days, ascitic fluid was collected from inoculated mouse. Lewis cells were collected after centrifugation and resuspended at $2 \times 10^{6} / \mathrm{mL}$, and then $0.2 \mathrm{ml}$ cell suspension was injected subcutaneously to the right armpit. 5 days after tumor implantation, the mice with tumor size of $0.3-0.5 \mathrm{~cm}$ in diameter were grouped randomly into 4 groups: control, Yangyin Fuzheng Decoction, cisplatin, and combination group, 8 mice per group. Drug treatment started on Day 5 after implantation with fasting overnight. $0.4 \mathrm{~mL}$ Yangyin Fuzheng Decoction was given to both Yangyin Fuzheng Decoction group and combination group intragastrically every day for 10 days. $0.2 \mathrm{~mL} 2$ $\mathrm{mg} / \mathrm{kg}$ cisplatin was administrated to both cisplatin and combination groups intragastrically every day for 10 days. The control group was administrated with equal amount of saline. Body weight, food intake, the activity and death of animal, skin color and defecation were monitored and recorded. 24 hours after the last administration, mice were weighted and euthanized. Tumor tissues were separated and weighted. Tumor growth inhibition rate was calculated using formula: Tumor growth inhibition rate $=$ (average tumor weight of control group - average tumor weight of experimental group) / average tumor weight of control group $\times 100 \%$.

\section{Immunohistochemical analysis}

Tumor tissue was fixed in 10\% formaldehyde for 24 hours, embedded in paraffin, and then sliced into 5 $\mu \mathrm{m}$ slides. Next, endogenous peroxidase activity in the slides was quenched and unspecific binding was blocked by incubation with $5 \%$ normal goat serum for $1 \mathrm{~h}$, then incubated with Bcl-2, Bax and p53 antibody (Boster, Wuhan, China) overnight at $4^{\circ} \mathrm{C}$. Next the slides were analyzed with Super Vision Two-step Immunohistochemistry Assay Kit (Boster, Wuhan, China) and incubated with diaminobenzidine. Images were captured using Motic3000 microscope imaging system and integral optical density (IOD) was qualified using Image-pro plus 6.0 system.

\section{Statistical analysis}

All experiment data were presented as average \pm standard error and analyzed using SPSS 18.0 software (SPSS Inc., Chicago, IL, USA). P < 0.05 was considered as statistical significance. 


\section{Results}

\section{Yangyin Fuzheng Decoction suppressed lung cancer growth in mice}

To investigate the anti-tumor effect of Yangyin Fuzheng Decoction, Lewis lung cancer cells were implanted into mice to form tumors, and the mice were treated with cisplatin, Yangyin Fuzheng Decoction, combination of cisplatin and Yangyin Fuzheng Decoction, or saline as control. As expected, cisplatin treatment effectively suppressed tumor growth by $57.5 \%$ in comparison with control on Day 21 (Fig. 1A, B). Yangyin Fuzheng Decoction treatment group showed significant tumor growth suppression by $52.4 \%$. Combination of cisplatin and Yangyin Fuzheng Decoction treatment group showed tumor growth suppression by $71.1 \%$, significantly higher than that in Yangyin Fuzheng Decoction or cisplatin treatment alone group $(\mathrm{P}<0.05$, Fig. $1 \mathrm{~B})$. These data indicated that Yangyin Fuzheng Decoction enhanced the anti-tumor efficacy of cisplatin.

Since Yangyin Fuzheng Decoction is used as health improving medicine in China, we also monitored body weight of mice as a measurement of general health during treatment. Cisplatin treated mice had significantly decreased body weight on Day 21 after treatment, while Yangyin Fuzheng Decoction treated mice gain more weight than control group. Combined treatment of Yangyin Fuzheng Decoction and cisplatin had lower body weight than control group but had significantly higher body weight than cisplatin group (Fig. 1C). These results suggested that Yangyin Fuzheng Decoction could alleviate the side effect and improve the health condition of mice during cancer chemotherapy.

\section{Yangyin Fuzheng Decoction promoted lung tumor cell death}

To investigate how Yangyin Fuzheng Decoction improved anti-tumor efficacy, tumor tissue samples were collected 21 days after the start of treatment. As shown in Fig. 2A, tumor tissues from control group were quite large, with irregular shape, fragile and easy to breakup, and very few necrotic areas were observed. Tumor tissues from Yangyin Fuzheng Decoction group were smaller than that from control group, had irregular shape, and some necrotic areas were observed. Tumors from cisplatin group were significantly smaller, had irregular shape, and many necrotic areas were observed. Combined treatment group had significant smaller tumors, irregular shape, and abundant necrotic areas. These results suggested that cisplatin and YFD could inhibit tumor growth through the induction of tumor cell death, and combined treatment with cisplatin and YFD further enhanced the effect.

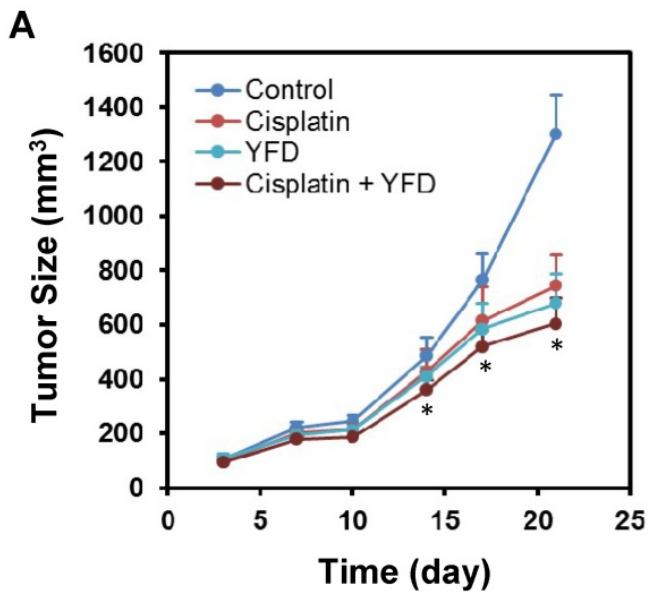

B

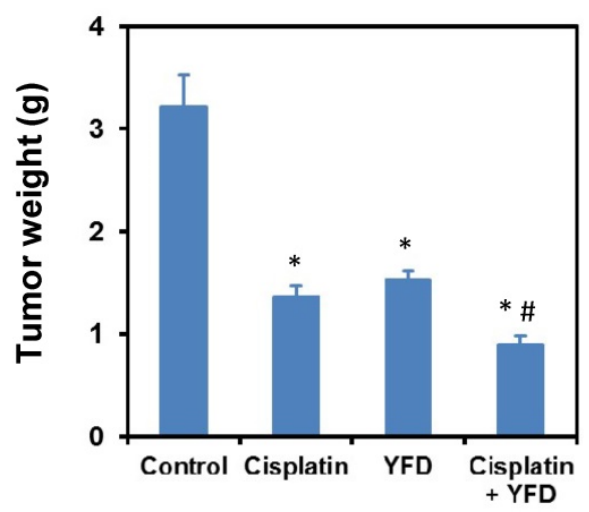

C

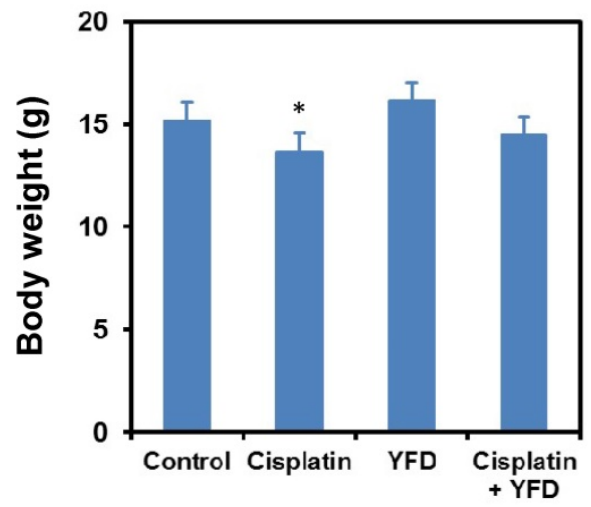

Figure 1. Lewis lung cancer growth in each group. A. C57BL/6 mice with Lewis lung tumor were treated with saline control, Yangyin Fuzheng Decoction (YFD), cisplatin, or combination of YFD and cisplatin for 10 days. The growth of tumor was monitored by caliper measurement of tumor size twice a week for 3 weeks. $* \mathrm{P}<0.05$ Cisplatin + YFD vs. Control group. B. The tumor weight of each group on day $21(n=6)$. *P $<0.05$ vs. Control group. \#P $<0.05$ vs. YFD group or cisplatin group. C. Mice body weight of each group on day $21(n=6) . * P<0.05$ vs. Control group.

The tumor tissue from all four groups were then sectioned and stained with $\mathrm{H} \& \mathrm{E}$ for pathological analysis. As shown in Fig. 2B, tumor cells in control group showed nested, tight distribution, fast growing. Tumor cells were tightly aligned, cell size was not uniform and cell shape was irregular. The nuclei were big, abnormal, deep staining with uneven 
distribution, pathological karyokinesis was common, and invasion of lymphocytes was rare in tumor tissue. Tumors in Yangyin Fuzheng Decoction group also had dense aligned tumor cells, but the density was lower than that in control group. Chromatin condensation and pathological karyokinesis were less. Parts of tumor was necrotic and infusion of inflammatory cells was observed. In cisplatin treated tumor tissues, tumor cell density was significantly decreased, cells were swelling and abnormal, pathological karyokinesis and pleomorphic cells were significantly decreased. Large necrotic areas and the infusion of inflammatory cells in tumor stroma were observed. These results demonstrated that cisplatin treatment effectively induced tumor cell death in tumor tissue. In combined treatment groups we found decreased tumor cell density, significantly decreased pathological karyokinesis and pleomorphic cells, large necrotic area and large amount of infusion lymphocytes. Collectively, these results indicated that Yangyin Fuzheng Decoction not only effectively induced tumor cell death, but also improved immune response to tumor cells.

\section{Yangyin Fuzheng Decoction modulated the expression of pro-apoptotic and anti-apoptotic proteins}

As a DNA damage inducing agent, cisplatin predominantly induces tumor cell death through apoptosis [2]. To investigate the mechanism by which Yangyin Fuzheng Decoction induced tumor cell death, we examined the expression levels of pro-apoptotic proteins 553 and Bax and anti-apoptotic protein Bcl-2 in tumor samples by immunohistochemical staining. Bcl-2 is a key protein on mitochondria membrane that protect cells from apoptosis by the inhibition of caspase activation through blocking of cytochrome $\mathrm{C}$ release from the mitochondria [12]. We found that Bcl-2 level in Yangyin Fuzheng Decoction or cisplatin group was significantly decreased about $25 \%$ in comparison with control group (Fig. 3A, B). In combined treatment group, Bcl-2 level was further decreased to about $45 \%$ of control group (Fig. 3A, B). One the other hand, Bax is a pro-apoptotic protein that mediates cytochrome $\mathrm{C}$ release in response to apoptotic signal, and p53 is an important tumor suppressor that function as a transcription factor to upregulate pro-apoptotic proteins and downregulate anti-apoptotic proteins [13]. The results showed that the expression of pro-apoptotic proteins p53 and Bax significantly increased more than 2-fold after cisplatin or YFD treatment $(\mathrm{P}<0.05$ vs. control group, Fig. 3C-F). Furthermore, p53 and Bax expression level in combined treatment group was the highest among all groups and was significant higher than that in cisplatin group $(\mathrm{P}<0.05$, Fig. 3D, F). Taken together, these results indicated that Yangyin Fuzheng Decoction could enhance apoptosis in Lewis lung cancer cells through the upregulation of pro-apoptotic proteins p53 and Bax and the downregulation of anti-apoptotic protein Bcl-2.

A

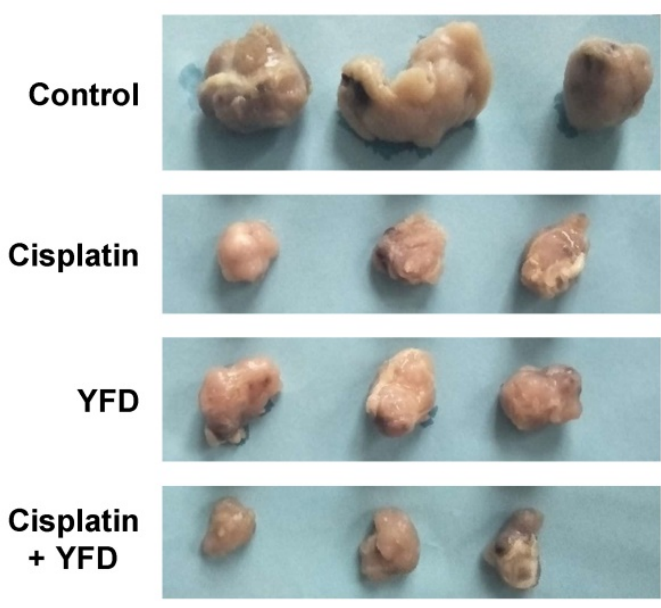

B
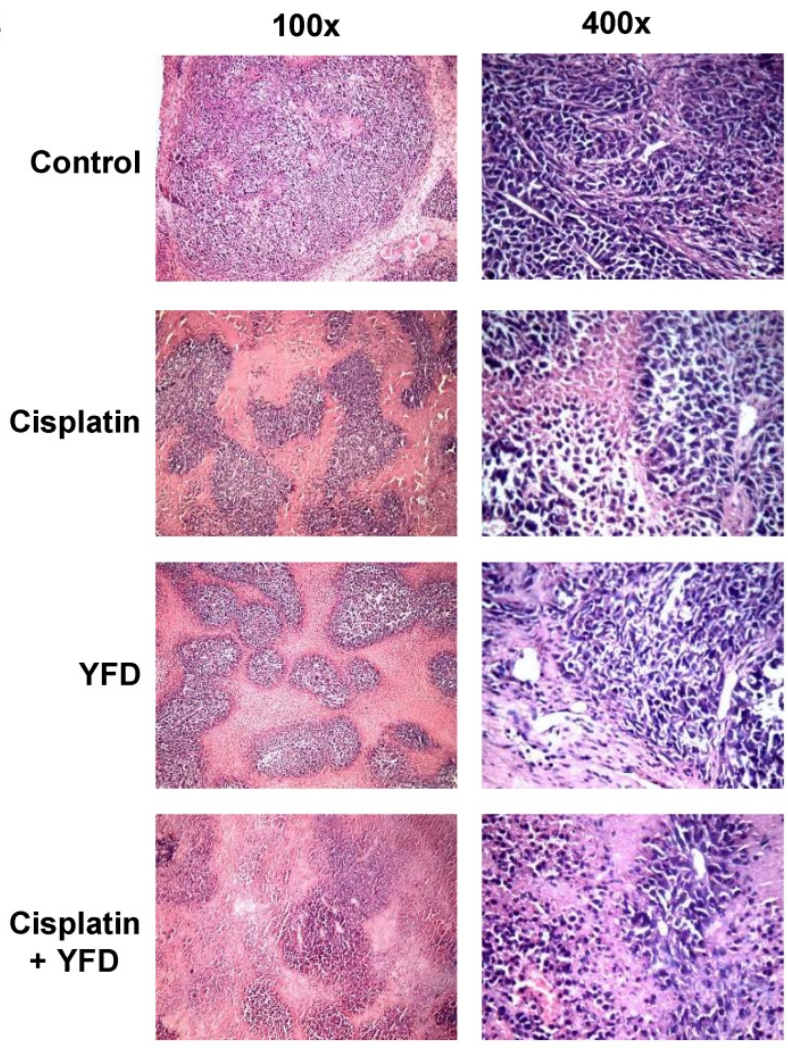

Figure 2. Pathology of Lewis lung tumor tissues in each group. A. The morphology of the tumor tissues from mice treated with saline (control), YFD, cisplatin and YFD plus cisplatin on Day 21. B. Hematoxylin eosin staining of the tumor tissues. $100 \times$ and $400 \times$ magnification. Shown were representative images from 8 mice in each group. 
A
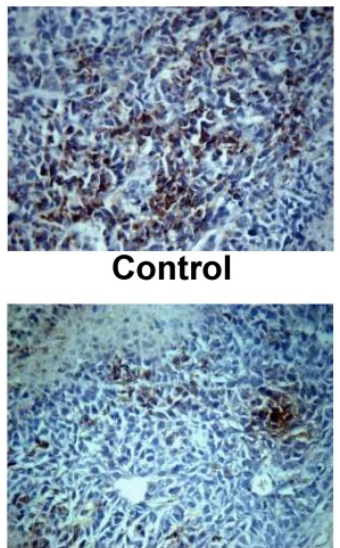

Cisplatin
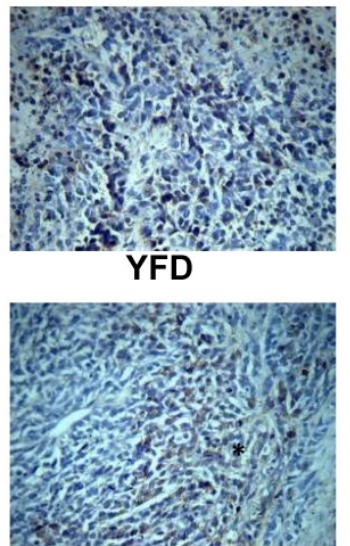

Cisplatin + YFD

B

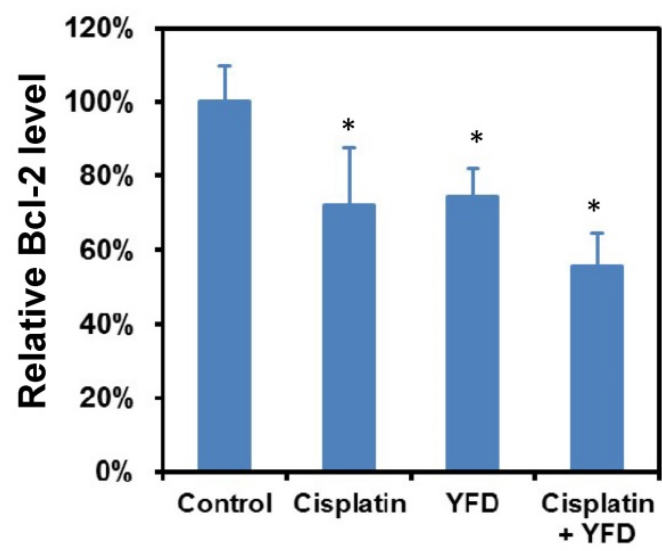

C

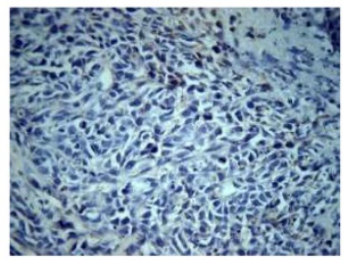

Control

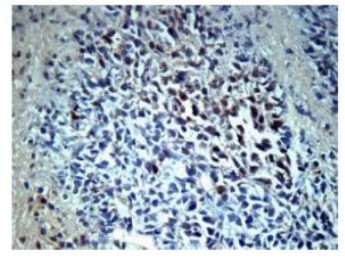

Cisplatin

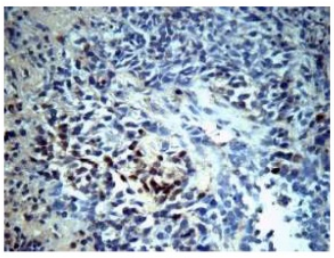

YFD

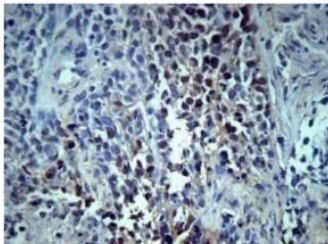

Cisplatin + YFD

D

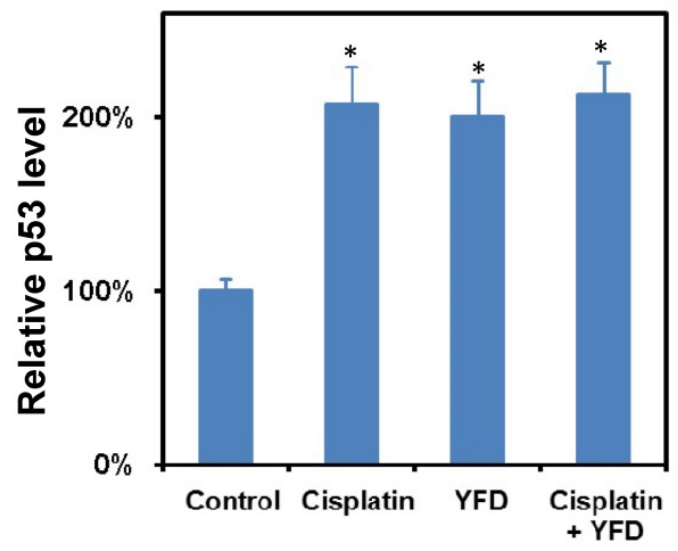

E

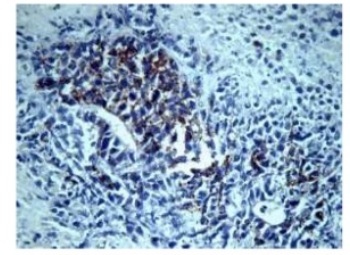

YFD
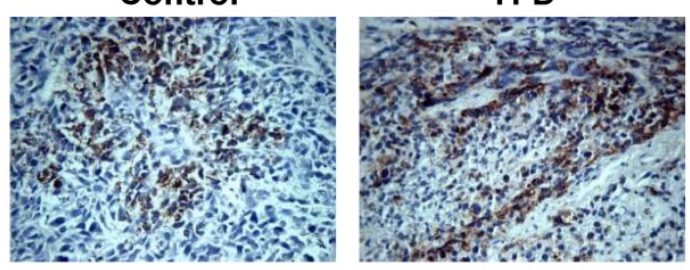

$\mathbf{F}$

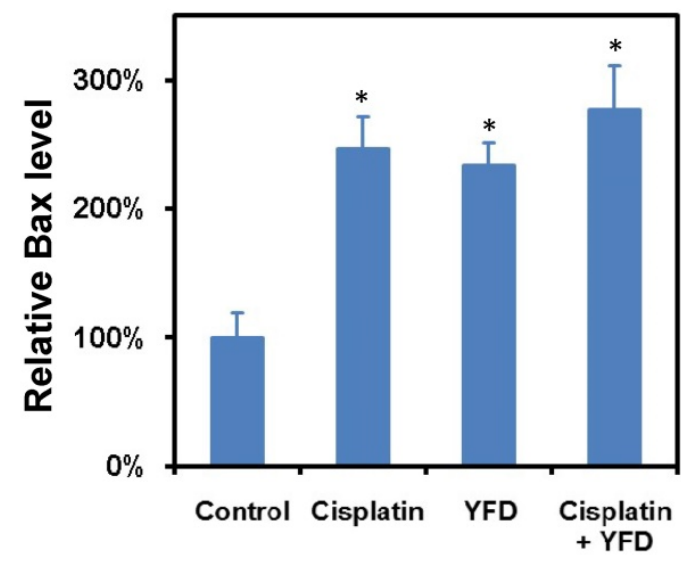

Cisplatin

Figure 3. Immunohistochemical analysis of $\mathrm{Bcl}-2$, Bax and $\mathrm{p} 53$ in Lewis lung tumor tissues in each group. A. Representative staining of Bcl-2 in tumor tissues from mice treated with saline (control), YFD, cisplatin and YFD plus cisplatin on Day 21.400x magnification. B. Semi-quantitative analysis of $B c l-2$ staining ( $n=6)$. $* P<0.05$ vs. Control group. C. Representative staining of $p 53$ in tumor tissues. $400 \times$ magnification. D. Semi-quantitative analysis of $p 53$ staining $(n=6)$. $* P<0.05$ vs. Control group. E. Representative staining of Bax in tumor tissues. $400 \times$ magnification. F. Semi-quantitative analysis of Bax staining $(n=6)$. *P $<0.05$ vs. Control group. 


\section{Discussion}

Traditional Chinese medicines have been widely used in cancer treatment in China not only for targeting tumor cells directly, but also for improving the health condition of patient in support of chemotherapy or radiotherapy [6]. Among them, SFJ is a traditional China medicine which has been applied to a variety of cancer treatment $[8,14]$. SFJ is a simple extract from two herbs Radix Astragali and Radix Codonopsis at 1:1 ratio. Traditionally, Radix Astragali is used to modulate immune system in the treatment of immunodeficiency disease, and Radix Codonopsis is used to treat dyspepsia, fatigue, bronchitis, cough and inflammation. SFJ has been shown in a serial of studies to effectively improve tumor suppression and reduce the toxicity of chemotherapy in a variety of cancers $[11,14,15]$. These results indicate that Radix Astragali and Radix Codonopsis are effective in support of cancer chemotherapy. In this study, we investigated the anti-tumor effect of a complex traditional Chinese medicine Yangyin Fuzheng Decoction (YFD). Our results demonstrated that YFD can inhibit tumor growth when administered alone and improve the heathy condition of experimental animals as indicated by increased body weight. Additionally, YFD can enhance anti-tumor effect of cisplatin.

To understand how YFD improved anti-tumor response, we performed pathology analysis on tumor tissue samples after treatment. Our results showed that YFD alone could cause necrosis in tumor tissues, and when combined with cisplatin, it led to more necrosis in tumor tissues than cisplatin alone. YFD treated tumor sample had decreased cell density and increased inflammatory cell invasion. In YFD/cisplatin combination treated group, the tumor cell density was further decreased and the invasion of inflammatory cell was more significant. These results indicate that YFD enhances anti-tumor effect by improving immune response to tumor tissues, consistent with the documented immune activation effect of Radix Astragali and Radix Codonopsis $[10,16]$. It is important to note that YFD is composed of 12 Chinese medicinal herbs. In addition to Radix codonopsis and Radix astragali, the other ten herbs include Radix glehniae, Gigeriae galli, Glycyrrhizae, Herba hedyotidis diffusae, Radix ophiopogonis, Pericarpium citri reticulatae, Radix angelicae sinensis, Poria, Rhizoma diosscoreae, and Radix asparagi. These herbs have been shown to improve the quality of life and reduce some adverse events caused by radiotherapy or chemotherapy in advanced oesophageal cancer patients [17]. Thus, YFD may have better efficacy than SFJ to be used on lung cancer patients with radiotherapy or chemotherapy.

Apoptosis is a major pathway by which anti-cancer drugs kill cancer cells. To understand the mechanism by which YFD induced Lewis lung cancer cell death, we performed immunohistochemical analysis of pro-/anti-apoptotic proteins p53, Bax and Bcl-2. Tumor suppressor p53 is a key transcription factor that plays a central role in apoptosis induction and its cellular level is under tight regulation [13]. p53 up-regulates a variety of pro-apoptotic proteins in both intrinsic and extrinsic apoptotic pathways, including Bax, PUMA and death receptors. At the same time, it downregulates anti-apoptotic proteins, such as Bcl-2 and survivin, to sensitize cells to the apoptosis signals. Induction of p53 would activates the cellular apoptotic pathways. Bax is the major pro-apoptotic factor in intrinsic apoptotic pathway that mediates the release of cytochrome $C$ from the mitochondria to form apoptosome [18]. On the contrary, Bcl-2 is the key anti-apoptotic protein that blocks the release of cytochrome $\mathrm{C}$ from the mitochondria $[13,19]$. Our results showed that Bcl-2 level decreased while Bax and p53 levels significantly increased in tumor tissues after the treatment with YFD, suggesting that YFD may directly kill tumor cells through the induction of apoptosis. Combined treatment with YFD and cisplatin caused further increased expression of p53 and Bax and decreased expression of Bcl-2, compared to YFD or cisplatin treatment alone. These results suggest that YFD and cisplatin can function synergistically to modulate Bax/Bcl-2 level to induce tumor cell apoptosis, similar to the synergistic action of ginsenoside $\mathrm{Rg} 3$ and paclitaxel in breast cancer [20].

In conclusion, Yangyin Fuzheng Decoction improved health condition of cisplatin treated mice and enhanced anti-tumor efficacy of cisplatin through inducing cancer cell apoptosis and improving immune response to cancer cells. Yangyin Fuzheng Decoction could be a promising adjunct agent for lung cancer chemotherapy.

\section{Acknowledgement}

This study was supported by Natural Science Fund of Heilongjiang Province (No. H2016095).

\section{Competing Interests}

The authors have declared that no competing interest exists.

\section{References}

[1] She J, Yang P, Hong Q, et al. Lung cancer in China: challenges and interventions. Chest 2013;143: 1117-26.

[2] D'Addario G, Pintilie M, Leighl NB, et al. Platinum-based versus non-platinum-based chemotherapy in advanced non-small-cell lung cancer: a meta-analysis of the published literature. J Clin Oncol 2005;23:2926-36. 
[3] Ramalingam S, Belani C. Systemic Chemotherapy for Advanced Non-Small Cell Lung Cancer: Recent Advances and Future Directions. Oncologist 2008;13:5-13.

[4] Dempke WC. Targeted Therapy for NSCLC -- A Double-edged Sword? Anticancer Res 2015;35: 2503-12.

[5] Li J, Wang JC, Ma B, et al. Shenqi Fuzheng Injection for advanced gastric cancer: a systematic review of randomized controlled trials. Chin J Integr Med 2015;21:71-9.

[6] Cao C, Han D, Su Y, et al. Ginkgo biloba exocarp extracts induces autophagy in Lewis lung cancer cells involving AMPK / mTOR / p70S6k signaling pathway. Biomed Pharmacother 2017;93:1128-35.

[7] Zhu X, Zhou Y, Xu Q, et al. Traditional Chinese medicine Jianpi Bushen therapy suppresses the onset of pre-metastatic niche in a murine model of spontaneous lung metastasis. Biomed Pharmacother 2017; 86:434-40.

[8] Dong J, Su SY, Wang MY, et al. Shenqi fuzheng, an injection concocted from Chinese medicinal herbs, combined with platinum-based chemotherapy for advanced non-small cell lung cancer: a systematic review. J Exp Clin Cancer Res 2010;29:137.

[9] Wang J, Tong X, Li P et al. Bioactive components on immuno-enhancement effects in the traditional Chinese medicine Shenqi Fuzheng Injection based on relevance analysis between chemical HPLC fingerprints and in vivo biological effects. J Ethnopharmacol 2014;155:405-15.

[10] Cao DD, Xu HL, He AB, et al. The Effect of ShenQi FuZheng Injection in Combination with Chemotherapy versus Chemotherapy Alone on the Improvement of Efficacy and Immune Function in Patients with Advanced Non-Small Cell Lung Cancer: A Meta-Analysis. PLoS One 2016;11:e0152270.

[11] Xu R, Lin L, Li Y, et al. ShenQi FuZheng Injection combined with chemotherapy in the treatment of colorectal cancer: A meta-analysis. PLoS One 2017;12:e0185254.

[12] Elmore S. Apoptosis: a review of programmed cell death. Toxicol Pathol 2007;35:495-516.

[13] Chen K, Zhang F, Ding J, et al. Histone Methyltransferase SETDB1 Promotes the Progression of Colorectal Cancer by Inhibiting the Expression of TP53. J Cancer 2017;8:3318-30.

[14] Dong BY, Wang C, Tan L, et al. Inhibitory effect of Shenqi Fuzheng injection combined with docetaxel on lung cancer cells. J. Zhejiang Univ Sci B 2017;18:76-8.

[15] Yu SZ, Yang GH, Feng JM. Clinical study on treatment of malignant neoplasms with Shenqi Fuzheng Injection and chemotherapy. Chin J Integr Med 2006; 4:84-6.

[16] $\mathrm{Du}$ J, Cheng BC, Fu XQ, et al. In vitro assays suggest Shenqi Fuzheng Injection has the potential to alter melanoma immune microenvironment. J Ethnopharmacol 2016;194:15-9.

[17] Chen X, Deng L, Jiang X, et al. Chinese herbal medicine for oesophageal cancer. Cochrane Database Syst Rev 2016;1:CD004520.

[18] Chipuk JE, Kuwana T, Bouchier-Hayes L, et al. Direct activation of Bax by p53 mediates mitochondrial membrane permeabilization and apoptosis. Science 2004;303:1010-4.

[19] Geng Y, Zhou Y, Wu S, et al. Sulforaphane Induced Apoptosis via Promotion of Mitochondrial Fusion and ERK1/2-Mediated 26S Proteasome Degradation of Novel Pro-survival Bim and Upregulation of Bax in Human Non-Small Cell Lung Cancer Cells. J Cancer 2017;8:2456-70.

[20] Yuan ZG, Jiang H, Zhu XH, et al. Ginsenoside Rg3 promotes cytotoxicity of Paclitaxel through inhibiting $\mathrm{NF}-\mathrm{kB}$ signaling and regulating $\mathrm{Bax} / \mathrm{Bcl}-2$ expression on triple-negative breast cancer. Biomed Pharmacother 2017;89:227-32. 